\title{
Manejo de la costra negra de la papa (Rhizoctonia solani) con el establecimiento de abonos verdes
}

\author{
Carlos A. Betancourth ${ }^{1 \star}$, Benjamín A. Sañudo², Carlos A. Flórez ${ }^{2}$ y Claudia E. Salazar ${ }^{1}$ \\ (1) Facultad de Ciencias Agrícolas, Dpto. de Producción y Sanidad vegetal, Univ. de Nariño. Pasto, Colombia \\ (correo-e: cbet70@yahoo.com; claudiasalazarg@udenar.edu.co). \\ (2) Grupo de investigación de Sanidad Vegetal (GRISAV), Univ. de Nariño. Pasto, Colombia \\ (correo-e: cflorezcasanova@outlook.com) \\ * Autor a quien debe ser dirigida la correspondencia
}

Recibido Oct. 27, 2020; Aceptado Dic. 28, 2020; Versión final Ene. 10, 2021, Publicado Abr. 2021

\section{Resumen}

El principal objetivo de esta investigación fue determinar el beneficio de las especies Avena sativa L. (avena), Hordeum vulgare L. (cebada), Sinapsis alba L. (mostaza) y Rhaphanus rhaphanistrum L. (nabo forrajero) como abonos verdes para controlar la incidencia de la costra negra y el cancro del tallo (Rhizoctonia solani Kühn) en la producción de papa Diacol Capiro. Se utilizó un diseño de bloques al azar con tres repeticiones. La incidencia de $R$. solani se determinó mediante observación de síntomas asociados a brotación tardía y enrollamiento foliar en las etapas de emergencia y tuberización. Se cuantificó el peso promedio de tubérculos grandes y medianos y el rendimiento comercial. Los resultados mostraron disminuciones en la enfermedad en aproximadamente $5 \%$ con los abonos verdes e incrementos de papa comercial sobre las 5 t. ha ${ }^{-1}$. Se concluye que todas las especies utilizadas como abonos verdes mostraron menor incidencia de la enfermedad e influyeron positivamente en los rendimientos de tubérculos, sobresaliendo el comportamiento de la cebada.

Palabras clave: biomasa aérea; cereales; crucíferas; biofumigación; fitopatógenos del suelo

\section{Management of the black scurf (Rhizoctonia solani) in potato by using green manure}

\begin{abstract}
The primary objective of this research study was to examine the possible benefits of using Avena sativa $\mathrm{L}$. (oat), Hordeum vulgare L. (barley), Sinapsis alba L. (yellow mustard), and Rhaphanus rhaphanistrum L. (radish) as green manures to control the incidence of black scurf and stem canker (Rhizoctonia solani Kühn) in Diacol Capiro potato. A randomized block design with three replications was used. The incidence of $R$. solani was determined by observing symptoms associated with late sprouting and foliar curl in the emergence and tuberization stages. The average weight of large and medium tubers per plant was quantified, as well as the commercial yield. The results showed decreases in disease incidence of approximately $5 \%$ and increases in commercial potatoes by over $5 t^{\text {. }}$ ha $^{-1}$. It is concluded that all the species, especially barley, that were used as green manure reduced the incidence of the black scurf and stem canker disease and positively influenced tuber yields.
\end{abstract}

Keywords: aerial biomass; cereals; crucifers; biofumigation; soil phytopathogens 


\section{INTRODUCCIÓN}

Es evidente la distribución e incidencia generalizada de la costra negra o rizoctoniasis causada por el hongo Rhizoctonia solani Kühn (Teleomorfo: Thanatephorus cucumeris) en el cultivo de papa en Nariño, tanto de las variantes de guata (Solanum tuberosum L.) como las de criolla amarilla (Solanum phureja Juz. y Bukasov), sin tener una información actualizada sobre la severidad y reacción particular de los genotipos cultivados en el departamento (Betancourth et al., 2020). La medida de manejo más popularizada es la aplicación de fungicidas sintéticos en la siembra y las primeras etapas de crecimiento de las plantas de papa, principalmente con el empleo de moléculas de los grupos químicos Thiazole Carboxanilida y Pirazol Carboximida, cuyo modo de actuación es similar, afectando la enzima succinato deshidrogenasa y por lo tanto bloqueando el proceso respiratorio del hongo (Xiong et al., 2015). Su uso frecuente por cerca de 15 años, posiblemente conlleva a la aparición de variantes de $R$. solani con características de resistencia cruzada a los productos con el mismo modo de actuación (Sierotzki, y Scalliet, 2013).

Ante tal situación, hay necesidad de evaluar otras medidas de manejo, integrando el empleo de cultivares más tolerantes, el uso adecuado de plaguicidas con distintos mecanismos de actuación y efectivos contra el fitopatógeno, como también productos conteniendo unidades reproductivas de microorganismos antagónicos, además de la rotación de cultivos, incluyendo la incorporación de especies vegetales como abonos verdes (Tsror, 2010; Yossen et al., 2011; Chimouriya et al., 2018). El establecimiento e incorporación de los abonos verdes en el suelo, evidentemente trae beneficios sobre la fertilidad natural de los suelos principalmente con un mejoramiento de las condiciones físicas, con la contribución de una microbiota heterótrofa con distintas funciones, conllevando a la formación de agregados que benefician la estructuración. También hay incremento de poblaciones que cumplen distintos procesos de antagonismo sobre hongos fitopatógenos habitantes naturales en el suelo, entre los cuales está Rhizoctonia solani (Larkin y Griffin, 2007; Ascencion et al., 2015).

Se ha demostrado que especies de la familia Brassicaceae poseen componentes naturales como los glucosinolatos, producidos a partir del metabolismo secundario de las plantas que al ser utilizadas como abonos verdes liberan una serie de compuestos tóxicos, en especial la capacidad biofumigante de los isotiocianatos, que limitan directamente el desarrollo hongos patógenos del suelo (Larkin y Griffin, 2007; Vandicke et al., 2020). Dayegamiye et al., (2017), exponen que la incorporación al suelo de cereales como variedades de rápido crecimiento de cebada, avena o trigo, cuyo aporte considerable de biomasa trae consigo efectos positivos sobre las propiedades del suelo y su microbiota; condiciones que predisponen una menor incidencia de fitopatógenos.

Esta investigación se realizó para determinar el efecto benéfico de los abonos verdes en la disminución de la incidencia de Rhizoctonia solani y mejoramiento de la producción de papa guata variedad Diacol Capiro en tres municipios de Nariño. Para ello, se evaluaron las especies de avena (Avena sativa L.), cebada (Hordeum vulgare L.), mostaza (Sinapsis alba L.) y nabo forrajero (Rhaphanus rhaphanistrum L.), sobre la incidencia de $R$. solani y respuestas de rendimiento comercial.

\section{MATERIALES Y MÉTODOS}

La investigación se realizó a partir de segundo semestre agrícola de 2019 con duración de un año, en los municipios de Pasto, Ipiales y Túquerres estableciendo pruebas en las veredas Mapachico Centro (2710

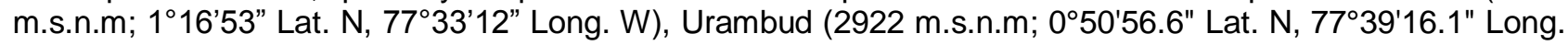
W) y Santa Ana (2843 m.s.n.m; 1 ${ }^{\circ} 5^{\prime} 3.22^{\prime \prime}$ Lat. N, 77³3'36.9" Long. W). En cada localidad se escogió un lote donde se había sembrado papa durante dos semestres y con la determinación de la presencia de costra negra en el último cultivo mediante la observación de síntomas de quemazón de estolones en estados iniciales de desarrollo y enrollamiento foliar para la etapa de floración de las plantas. La incidencia de la afectación fue de 35,18 y $42 \%$, respectivamente para las tres localidades.

\section{Establecimiento e incorporación de abonos verdes}

Durante el segundo semestre de 2019 , en cada lote debidamente preparado con un área de $24 \times 17 \mathrm{~m}$, se trazaron tres bloques de $24 \mathrm{~m}$ de largo y $5 \mathrm{~m}$ de ancho, con separación entre ellos de un metro. Por bloque se hizo la demarcación de cinco parcelas de $4 \times 5 \mathrm{~m}$ con separación entre ellas por calles de $1 \mathrm{~m}$; donde se estableció un diseño experimental en distribución de bloques al azar con cinco tratamientos y tres repeticiones. Los tratamientos corresponden a un testigo y la siembra al voleo de semilla de avena, cebada, mostaza y nabo, en cantidad de $70 \mathrm{~kg}$. ha-1 para las gramíneas y $50 \mathrm{~kg}$. ha-1 de las crucíferas. Al momento de la siembra se hizo una fertilización también al voleo con fertilizante 13-26-6 en cantidad de $200 \mathrm{~kg}$. ha- ${ }^{-1}$, para hacer luego un tapado manual empleando rastrillo. Después de un mes se realizó una deshierba a mano en cada una de las parcelas, más la aspersión del insecticida Dimetoato $1.5 \mathrm{cc} / \mathrm{L}$ de agua para el control de insectos chupadores. 
En la etapa de espigamiento y antesis de las gramíneas, como de floración total de las crucíferas se hizo el corte y picado de la biomasa aérea de los abonos verdes, con ayuda de una guadaña, determinando la cantidad de tejidos picados por metro cuadrado en un sitio representativo, después de cinco días, en cada parcela se realizó la incorporación de la biomasa mediante un picado uniforme con la ayuda de un azadón, procurando profundizar los tejidos a $20 \mathrm{~cm}$ de la superficie (Prager et al., 2008).

\section{Establecimiento y manejo del cultivo de papa}

Después de dos meses de la incorporación de los abonos verdes, se realizó la delimitación del área, conservando la misma distribución experimental en el diseño de bloques al azar con cinco tratamientos y tres repeticiones, marcando bien las parcelas con su área de 4x5m. En cada parcela se hizo la preparación manual y el trazado de seis surcos de $4 \mathrm{~m}$ de longitud y con separación entre ellos de $0.80 \mathrm{~m}$. Posteriormente se realizó el guachado y el picado del fondo de los surcos para la siembra y fertilización de la papa, utilizando semilla de segunda $(60-80 \mathrm{~g})$ de papa Diacol Capiro, sembrando 11 tubérculos por surco a una distancia de $0.40 \mathrm{~m}$ entre sitios.

Al mes de la siembra, cuando se observó emergencia total de la papa, se realizó el abonamiento en franjas a lado y lado de las plantas, con abono 13-26-6 en cantidad de $500 \mathrm{~kg}^{\text {. ha-1 }}$, efectuando un picado uniforme para incorporar el fertilizante y aflojar el terreno a profundidad. Posteriormente, en la etapa de formación de tallos aéreos, se hizo una segunda fertilización con 15-15-15 en la misma cantidad y distribuyendo el fertilizante sobre el surco, para luego efectuar el picado de las calles y un primer aporque, realizándose un segundo aporque luego de un mes.

Cada 10 días después de la emergencia total, se hizo una rotación entre Fosetil aluminio $3.0 \mathrm{~g} / \mathrm{L}$ de agua, Propamocarb $2 \mathrm{cc} / \mathrm{L}$ de agua y Dimetomorfh $2.0 \mathrm{~g} / \mathrm{L}$ de agua para el control de gota (Phytophthora infestans). Conjuntamente se empleó la mezcla comercial Tiametoxan más Lambdacihalotrina $1.0 \mathrm{cc} / \mathrm{L}$ de agua, en rotación con Dimetoato $1.5 \mathrm{cc}$ por litro, Thyociclan Hidrogenoxalato $1.5 \mathrm{cc} / \mathrm{L}$ de agua y Metomyl $20 \mathrm{cc} / \mathrm{L}$ de agua, para el control de plagas de la parte aérea y subterránea, principalmente hacia el gusano blanco (Premnotrypes vorax), las polillas de la papa (Tecia solanivora y Phthorimaea operculella), Chisa (Ancognatha scarabaeoides y Phyllophaga obsoleta), mosca blanca (Trialeurodes vaporariorum) y trips (Frankliniella tuberosi y Thrips palmi).

\section{Incidencia de Rhizoctonia solani}

Para la etapa de emergencia total, se hizo la evaluación de las plantas emergidas a los 30 días después de la siembra, en los tres surcos centrales de cada parcela experimental $\left(12 \mathrm{~m}^{2}\right)$, en los sitios con brotación tardía se observó síntomas de quemazón y muerte de brotes. Luego en la etapa de inicio de tuberización, coincidiendo con la floración, en los mismos surcos se determinó el número de plantas con enrollamiento foliar, coincidiendo con chancros basales y necrosis de extremos de estolones y de raíces (Tsror, 2010; Bienkowski et al., 2010). Para comprobar la presencia de $R$. solani en los tejidos afectados se realizaron aislamientos en PDA siguiendo el protocolo sugerido por Carling y Leiner (1986).

\section{Producción de tubérculos}

En los surcos centrales de cada parcela y en la época de cosecha, se realizó la colecta de tubérculos de cada planta, dejando la producción por sitio para hacer la evaluación de acuerdo con la siguiente escala, que consiste de cuatro categorías: 1) Producción normal de tubérculos en su mayoría de tamaño grande y mediano; 2) Producción media con una reducción del 50 \% respecto a la producción normal; 3) Producción escasa con una reducción del $75 \%$ respecto a la producción normal, en su mayoría de tubérculos pequeños; y 4) Producción muy escasa de tubérculos grandes, medianos, pequeños o deformes. Con la escala descrita se hizo la corroboración de la evaluación de incidencia en plantas por $R$. solani. Posteriormente se realizó la separación de tubérculos grandes, medianos y pequeños, para hacer el conteo y pesaje de cada categoría, obteniendo la producción de tubérculos por planta en relación a los sitios cosechados. Con la producción conjunto de tubérculos grandes y medianos, se obtuvo la producción por hectárea de papa comercial en toneladas, teniendo en cuenta las distancias de siembra y la población de plantas.

La interpretación estadística se realizó de manera manual, con los datos cuantitativos de incidencia de $R$. solani en las etapas de emergencia y tuberización, así como para los valores de rendimiento, los cuales se procesaron mediante análisis de varianza y la comparación de medias con la prueba de Tukey (a0.05), de acuerdo con una distribución combinada de localidades (factor a) y tratamientos (factor b) en base al diseño de bloques al azar, con el modelo matemático:

$$
Y i j k=\mu+A j+B k(j)+T i+(T A) i j+e i j k .
$$




\section{RESULTADOS}

Para determinar la incidencia de Rhizoctonia solani se evidenciaron síntomas de afectación en las etapas del cultivo de emergencia y tuberización. En la etapa de emergencia se observó lesiones en la base de los tallos en brotación ocasionando secamientos parciales y debilitamiento en el desarrollo; tanto en tallos, estolones y raíces; las lesiones evidenciadas tenían aspecto seco, de color marrón y generalmente hundidas (Figura 1).

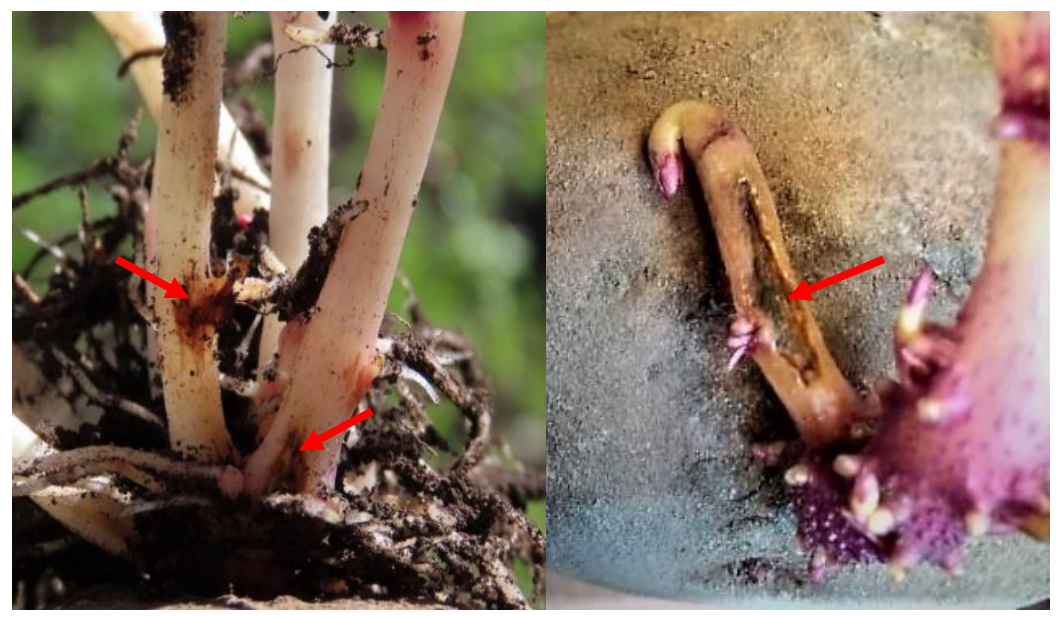

Fig. 1: Ataques tempranos de Rhizoctonia solani en estolones y brotes

\section{Incidencia de R. solani en etapas de emergencia y tuberización}

En el análisis de varianza de la Tabla 1, para la variable de incidencia de $R$. solani en etapa de emergencia se observaron diferencias estadísticas entre localidades y tratamientos; no se presentaron diferencias significativas para la interacción localidades por tratamientos. (En la tabla, * indica diferencias significativas y ns indica diferencias no significativas).

Tabla 1: ANOVA para los porcentajes de incidencia de Rhizoctonia solani en etapas de emergencia y tuberización de papa Diacol Capiro después de la inclusión de abonos verdes.

\begin{tabular}{|l|c|c|c|c|}
\hline \multicolumn{1}{|c|}{ Fv } & Gl & \multicolumn{2}{c|}{ Cuadrados medios } & F tabla \\
\hline & & Incidencia en emergencia & Incidencia en tuberización & \\
\hline Localidades (a) & 2 & $31.74^{*}$ & $12.51^{*}$ & 5.4 \\
\hline Tratamientos (b) & 4 & $71.68^{*}$ & $75.82^{*}$ & 2.78 \\
\hline Localidades x Tratamientos & 8 & $2.52^{\mathrm{ns}}$ & $0.21^{\mathrm{ns}}$ & 2.36 \\
\hline Error & 24 & 1.72 & 0.77 & \\
\hline
\end{tabular}

En la Tabla 2, se determinaron mayores ataques en la etapa de emergencia en el municipio de Túquerres con $10.63 \%$ de afectación promedio y diferencias significativas en relación a las localidades de Ipiales y Pasto con menos del $10 \%$ de incidencia de acuerdo con la disposición de los propágulos del hongo en el suelo. Además, se observa similar comportamiento de los abonos verdes, presentando ataques entre el 7.43 a $8.20 \%$ con diferencias significativas respecto al testigo (14.19\%). (En la tabla, letras iguales indican diferencias no significativas).

Según la Tabla 1, para la variable de incidencia en etapa de tuberización se observan diferencias significativas entre localidades y tratamientos de manera independiente, pero no para la interacción, lo cual probablemente demuestra que el efecto patogénico está más relacionado con la capacidad de dispersión de $R$. solani además de las condiciones fisicoquímicas de los suelos y la variabilidad climática. Para esta variable se determina que con el tiempo hay incremento de inóculo en las áreas con mayor dispersión del patógeno como Pasto y Túquerres, donde la incidencia es mayor del $11 \%$, para diferencias significativas respecto a Ipiales con menos de $10 \%$ de afectación (Tabla 2). Respecto a los tratamientos se determinó que el testigo presento mayor afectación en cuanto al síntoma de enrollamiento foliar con más del $15 \%$, valor estadísticamente diferente a los obtenidos con el empleo de abonos verdes. El mayor efecto depresor sobre el fitopatógeno se logró con el empleo de cebada, que solo permitió un $8.57 \%$ de enrollamiento foliar. 
Tabla 2: Comparación de porcentajes de incidencia de Rhizoctonia solani en las etapas de emergencia y tuberización en papa Diacol Capiro, después del establecimiento de abonos verdes en tres municipios de Nariño.

\begin{tabular}{|c|c|c|c|c|c|c|}
\hline \multicolumn{7}{|c|}{ Incidencia en emergencia } \\
\hline & \multicolumn{5}{|c|}{ Tratamientos (b) } & \multirow[b]{2}{*}{ Promedio a } \\
\hline Localidades (a) & Avena & Cebada & Mostaza & Nabo & Testigo & \\
\hline Pasto & 9.03 & 8.83 & 5.83 & 11.65 & 14.33 & $9.16^{b}$ \\
\hline Ipiales & 5.98 & 7.2 & 6.73 & 6.53 & 12.2 & $7.73^{b}$ \\
\hline Túquerres & 9.58 & 7.48 & 9.73 & 10.3 & 16.03 & $10.63^{a}$ \\
\hline Promedio b & $8.20^{b}$ & $7.83^{b}$ & $7.43^{b}$ & $8.20^{b}$ & $14.19^{a}$ & \\
\hline Tukey a $0.05=1.45$ & \multicolumn{6}{|c|}{ Tukey b $0.05=2.21$} \\
\hline \multicolumn{7}{|c|}{ Incidencia en tuberización } \\
\hline & \multicolumn{5}{|c|}{ Tratamientos (b) } & \\
\hline Localidades (a) & Avena & Cebada & Mostaza & Nabo & Testigo & Promedio a \\
\hline Pasto & 10.43 & 8.43 & 11.67 & 10.57 & 16.83 & $11.92^{a}$ \\
\hline Ipiales & 8.7 & 7.8 & 8.17 & 9.87 & 14.77 & $9.92^{b}$ \\
\hline Túquerres & 11.2 & 9.47 & 9.73 & 10.43 & 16.1 & $11.40^{a}$ \\
\hline Promedio b & $10.11^{b}$ & $8.57^{c}$ & $9.96^{b}$ & $10.28^{b}$ & $15.92^{a}$ & \\
\hline Tukey a $0.05=0.80$ & \multicolumn{5}{|c|}{ Tukey $b 0.05=1.22$} & \\
\hline
\end{tabular}

\section{Rendimiento de tubérculos por planta}

Los resultados del análisis de varianza para la variable peso promedio en kilos de tubérculos grandes y medianos por planta, permitieron determinar diferencias significativas entre localidades y tratamientos, como para su interacción (Tabla.3). (En la tabla, ${ }^{*}$ indica diferencias significativas y ${ }^{\text {ns }}$ indica diferencias no significativas).

Tabla 3: ANOVA para el peso de tubérculos grandes y medianos por planta $(\mathrm{kg})$ y rendimiento comercial (t. ha-1) de papa Diacol Capiro después de la inclusión de abonos verdes.

\begin{tabular}{|l|c|c|c|c|c|}
\hline & Gl & \multicolumn{3}{c|}{ Cuadrados medios } & F tabla \\
\hline & & $\begin{array}{c}\text { Tubérculos } \\
\text { Grandes }\end{array}$ & $\begin{array}{c}\text { Tubérculos } \\
\text { Medianos }\end{array}$ & $\begin{array}{c}\text { Rendimiento } \\
\text { comercial }\end{array}$ & \\
\hline Localidades (a) & 2 & $0.009^{*}$ & $0.003^{*}$ & 2.569 ns & 5.4 \\
\hline Tratamientos (b) & 4 & $0.050 *$ & $0.023^{*}$ & $58.046^{*}$ & 2.78 \\
\hline Localidades x Tratamientos & 8 & $0.002^{*}$ & $0.0003^{*}$ & $13.764^{*}$ & 2.36 \\
\hline Error & 24 & 1.72 & 0.77 & & \\
\hline
\end{tabular}

\section{Tubérculos grandes}

Los menores rendimientos de tubérculos grandes en peso se observan en Túquerres con diferencias significativas respecto a las otras dos localidades (Tabla 4), debido a una mayor incidencia de $R$. solani. Sin embargo, este efecto también puede llegar a ser influido por las condiciones físicas del suelo donde se realizó el ensayo, especialmente su textura franco arcillosa y la menor profundidad efectiva en el perfil. Todos los abonos verdes mostraron diferencias significativas respecto al testigo (Tabla 4). Al analizar la interacción, se puede determinar el efecto benéfico de la cebada en los tres ambientes permitiendo de manera general mayores pesos de tubérculos grandes, lo cual está confirmando el efecto supresor sobre $R$. solani al observar la incidencia del patógeno en las dos etapas de evaluación. (En la tabla, letras iguales indican diferencias no significativas).

\section{Tubérculos medianos}

De acuerdo con el análisis de varianza de la Tabla 3, el empleo y beneficio de abonos verdes permitió mayor rendimiento de tubérculos medianos por planta en relación con el testigo, presentando diferencias significativas para localidades, tratamientos y su interacción; no obstante, no se determinaron diferencias estadísticas entre las cuatro especies evaluadas como abonos verdes. 
Tabla 4: Comparación de pesos promedios de tubérculos por planta $(\mathrm{kg})$ y rendimiento comercial (t. ha $\left.{ }^{-1}\right)$ de papa Diacol Capiro, después de la incorporación de abonos verdes en tres municipios de Nariño para el manejo de Rhizoctonia solani.

\begin{tabular}{|c|c|c|c|c|c|c|}
\hline \multicolumn{7}{|l|}{ Tubérculos grandes } \\
\hline & \multicolumn{5}{|c|}{ Tratamientos (b) } & \multirow[b]{2}{*}{ Promedio a } \\
\hline Localidades (a) & Avena & Cebada & Mostaza & Nabo & Testigo & \\
\hline Pasto & $0.573^{b}$ & $0.652^{b}$ & $0.602 \mathrm{ab}$ & $0.563^{b}$ & $0.431^{c}$ & $0.564^{a}$ \\
\hline Ipiales & $0.573^{b}$ & $0.661^{a}$ & $0.620 \mathrm{ab}$ & $0.579^{b}$ & $0.420^{c}$ & $0.571^{a}$ \\
\hline Túquerres & $0.523^{a}$ & $0.575^{a}$ & $0.546^{a}$ & $0.543^{a}$ & $0.444^{b}$ & $0.526^{b}$ \\
\hline Promedio b & $0.556^{d}$ & $0.629^{a}$ & $0.589 \mathrm{bc}$ & $0.562^{\mathrm{cd}}$ & $0.432^{\mathrm{e}}$ & \\
\hline Tukey a $0.05=0.020$ & \multicolumn{2}{|c|}{ Tukey b $0.05=0.031$} & \multicolumn{3}{|c|}{ Tukey $a \times b=0.05=0.054$} & \\
\hline \multicolumn{7}{|l|}{ Tubérculos medianos } \\
\hline & \multicolumn{5}{|c|}{ Tratamientos (b) } & \\
\hline Localidades (a) & Avena & Cebada & Mostaza & Nabo & Testigo & Promedio a \\
\hline Pasto & 0.357 & 0.405 & 0.42 & 0.427 & 0.269 & 0.376 \\
\hline Ipiales & 0.412 & 0.454 & 0.375 & 0.414 & 0.346 & 0.4 \\
\hline Túquerres & 0.413 & 0.443 & 0.412 & 0.437 & 0.31 & 0.403 \\
\hline Promedio $\mathrm{b}$ & $0.393^{a}$ & $0.434^{a}$ & $0.402^{a}$ & $0.426^{a}$ & $0.308^{b}$ & \\
\hline \multicolumn{7}{|l|}{ Tukey $b 0.05=0.062$} \\
\hline \multicolumn{7}{|l|}{ Rendimiento comercial } \\
\hline & \multicolumn{5}{|c|}{ Tratamientos (b) } & \\
\hline Localidades (a) & Avena & Cebada & Mostaza & Nabo & Testigo & Promedio a \\
\hline Pasto & $20.947^{a b}$ & $23.316^{a}$ & $22.944^{a}$ & $22.275^{\mathrm{a}}$ & $17.299^{b}$ & 21.436 \\
\hline Ipiales & $22.200^{a}$ & $25.080^{a}$ & $22.380^{a}$ & $22.350^{a}$ & $17.076^{b}$ & 21.87 \\
\hline Túquerres & $22.210^{a}$ & $22.746^{a}$ & $21.355^{a}$ & $21.709^{a}$ & $16.932^{b}$ & 20.99 \\
\hline Promedio b & $21.786^{\mathrm{a}}$ & $23.847^{a}$ & $22.226^{a}$ & $22.112^{a}$ & $17.102^{b}$ & \\
\hline \multicolumn{7}{|c|}{ Tukey $a \times b 0.05=3.832$} \\
\hline
\end{tabular}

\section{Rendimiento comercial}

De acuerdo con el análisis de varianza de la Tabla 3, se determinan diferencias significativas entre tratamientos (abonos verdes versus testigo) y para la interacción localidades por tratamientos. No se presentan diferencias significativas entre localidades. En la tabla 4 se comparan los promedios de rendimiento en toneladas por hectárea, observándose, que en todos los ambientes y de manera general, el empleo de los abonos verdes permitió diferencias significativas respecto al testigo, con la situación particular de la avena en Pasto que no difirió con el testigo, debido a su menor aporte de biomasa aérea, por menor adaptación de la variedad Cayuse a las condiciones ambientales del sector. Por efectos de la incorporación de abonos verdes, se observa incrementos en rendimientos respecto al testigo, mayores hasta en cinco toneladas de papa por hectárea, siendo la cebada el mejor tratamiento, así no existan diferencias estadísticamente significativas respecto a las otras especies.

\section{DISCUSIÓN}

De acuerdo con las observaciones de campo, se determinó la presencia del patógeno, mediante las manifestaciones correspondientes a brotación tardía y escaso número de brotes, como también enrollamiento foliar en la etapa de floración de las plantas, lo cual está asociado respectivamente a la presencia de chancros necróticos en la base de los tallos principales cerca de la parte radicular, como a la pudrición apical de raicillas y estolones, esta característica multisintomática se puede deber a su variabilidad genética (Ferrucho et al., 2013; Kumar et al., 2017). En la etapa de floración, se incrementa la actuación de $R$. solani sobre los tejidos subterráneos más jóvenes como son las partes apicales de raicillas y estolones, ocasionando interferencias metabólicas vía haces vasculares, lo que en consecuencia conduce a enrollamiento parcial o total de las ramas foliares (Bienkowski et al., 2010; Kumar et al., 2017) (Figura 2). 


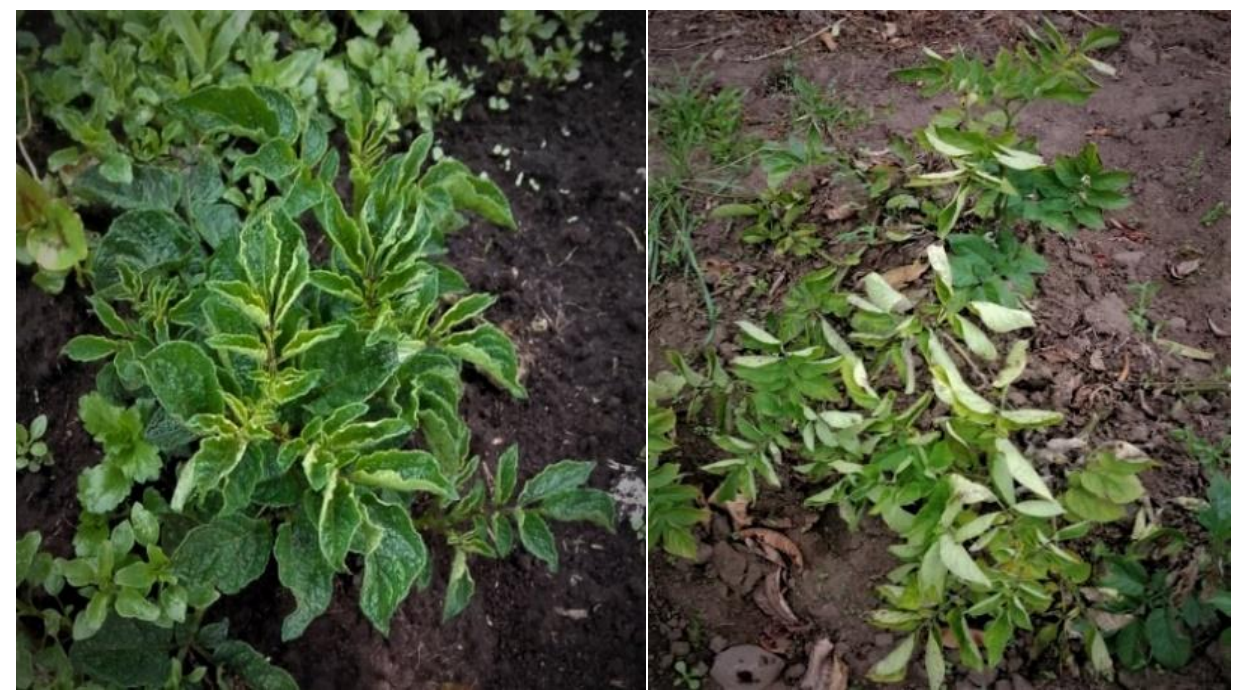

Fig. 2: Síntomas de enrollamiento parcial o total de las ramas foliares causados por Rhizoctonia solani.

La localidad de Ipiales presentó el menor valor de afectación probablemente debido a que el terreno de esta localidad, tiene un suelo liviano con textura franco arenosa, conduciendo a menor retención de humedad, lo que desfavorece la dispersión y colonización del patógeno (Tsror, 2010). El efecto positivo de los abonos verdes para disminuir la incidencia de $R$. solani se debe a la biomasa incorporada en los suelos, la cual es sometida a la acción de la microbiota heterótrofa gracias a una batería enzimática múltiple y complementaria para la mineralización de la materia orgánica, con obtención de fuentes carbonadas de energía y liberación de nutrientes, lo que permite incremento poblacional de rizobacterias y hongos, que en conjunto presentan características antagónicas con distintos efectos, lo cual puede llevar a la supresividad sobre $R$. solani (Larkin y Griffin, 2007; Handiseni et al., 2016; De Corato, 2020).

De acuerdo a lo expuesto por autores como Manici et al., (2004) y Mazzola (2010), dentro de la microbiota antagónica favorecida se encuentran bacterias como Bacillus, Pseudomonas y especies no patogénicas de Streptomyces, así como el hongo Trichoderma (Yossen et al., 2011), del hay especies con mayor tolerancia frente a isotiocianatos producidos por tejidos incorporados de Brassica, respecto a otros hongos patógenos del suelo (Larkin y Griffin, 2007; Vandicke et al., 2020). En este grupo de plantas, se ha identificado especies de mostaza como Brassica juncea y $B$. nigra poseen efecto biofumigante contra aislados del patógeno $R$. solani en papa y hortalizas (Rubayet et al., 2018; Abdallah, Yehia, y Kandil, 2020). Además, es probable que en la biodegradación de los residuos orgánicos haya liberación de metabolitos secundarios, los cuales podrían llegar a ser aprovechados por las plantas vía absorción radicular hacia la inducción de defensas contra $R$. solani (Soldevilla, 2009).

El mayor efecto benéfico de la cebada podría deberse a causa de la cantidad más alta de biomasa aportada (más de 50 toneladas por hectárea), la cual está constituida por tejidos más fibrosos, que permite mayor tiempo de actuación para la microbiota heterótrofa, lo que llega a favorecer la persistencia de las poblaciones con características de antagonismo o competencia por recursos (disponibilidad de energía, agua y nutrientes) afectando en diferente grado la capacidad de persistencia y colonización de $R$. solani (Parkinson y Coleman, 1991; Vukicevich et al., 2016). Por otra parte, es probable que haya producción de sustancias orgánicas específicas y con propiedades biocidas o biostáticas sobre el patógeno o con efecto positivo para el sistema radicular de la papa hacia respuestas defensivas (Larkin, 2013).

Los resultados respecto a las cantidades de tubérculos indican que en la etapa de tuberización hay influencia de las condiciones medioambientales de los sectores paperos sobre el crecimiento y producción de las plantas, considerándose también que la capacidad de retención de humedad de los suelos puede predisponer la persistencia del patógeno (Gonzáles et al., 2006). Se presentó mayor cantidad tubérculos grandes en la localidad de Ipiales con diferencias significativas en comparación con Pasto y Túquerres, debido a la menor proporción de inóculo del patógeno en el suelo y a que las condiciones físicas, favorecen un mejor desarrollo subterráneo de las plantas (Tsror y Perezt, 2005).

Los resultados respecto al rendimiento de tubérculos de segunda permiten suponer con confiabilidad, que el uso de abonos verdes, es una medida favorable para esta variable, porque las conversiones bioquímicas que ocurren en el suelo con la biomas aérea aportada, llega a favorecer una actividad efectiva en la rizosfera de las plantas de papa, con el aporte nutricional y de principios activadores de crecimiento, además de los procesos antagónicos que desfavorecen el establecimiento del agente causal de la costra negra (Bonanomi 
et al., 2020). Igualmente, se determina que la cebada como abono verde favorece un mejor crecimiento de la papa, respecto a avena y nabo con diferencias significativas, probablemente por el menor aporte de biomasa aérea de la primera especie o por la liberación de principios alelopáticos del nabo, lo cual debe comprobarse, aunque investigaciones han demostrado el uso de especies de nabo para el manejo integrado de enfermedades transmitidas por el suelo para mejorar el rendimiento de la papa (Khiareddine, 2016).

Aunque todas las especies utilizadas como abonos verdes demostraron el efecto benéfico del establecimiento e incorporación de biomasa aérea, para disminuir el ataque de $R$. solani, gracias a que influyen favorablemente en el establecimiento de poblaciones microbianas con capacidad antagónica, especialmente la cebada por su inherente composición fibrosa. Ello se observa de manera similar en los municipios de Pasto e Ipiales, pero en Túquerres, con mayor incidencia del patógeno, todos los abonos verdes ejercen efecto benéfico similar, siendo más notable el incremento de especies microbianas benéficas, con principios antagónicos inducibles ante la presencia R. solani (Ramírez et al., 2011; Larkin y Halloran, 2014; Vukicevich et al., 2016).

Los residuos vegetales incorporados al suelo traen con sigo cambios benéficos en el sistema suelo al presentar un horizonte superficial de mayor fertilidad, al suponer un equilibrio en sus características físicas permitiendo una adecuada aireación y contenido de humedad, lo que predispone un entorno beneficioso para el desarrollo de hábitat funcionales, que contribuyen para la producción y protección de los cultivos (Sampaio, 2009). La bondad de esta práctica abarca un mejoramiento integrado de la fertilidad natural de los suelos, especialmente en relación con las condiciones físicas y biológicas. En este último aspecto se considera que el valor biológico de los abonos verdes es el incremento de poblaciones microbianas heterótrofas, que conducen a una actividad bioquímica favorable para las plantas, mediante la producción de principios químicos activadores de crecimiento, como diferentes relaciones de antagonismo sobre microorganismos patógenos habitantes naturales del suelo (Smith et al., 2011; Larkin, 2013; De Corato, 2020).

El rendimiento mayor de tubérculos medianos que se logró con la práctica en todas las localidades, necesariamente debe conducir a aumentos en la producción comercial. Este beneficio de los abonos verdes es múltiple, en lo que respecta a procesos de bioconversión, tanto de la mineralización de la materia orgánica, como en la humificación de los suelos. En este camino, actúa una microbiota compleja, desde la degradación de los residuos orgánicos, siguiendo con la mineralización y asimilación de nutrientes, la oxidorreducción, la inmovilización de nutrientes y el equilibrio. En este último aspecto y gracias a la incorporación de tejidos vegetales, se genera una secuencia de poblaciones con distintos efectos antagónicos sobre patógenos, como es la competencia por espacio y nutrientes, la producción de metabolitos antibióticos y la acción parasitaria principalmente (Parkinson y Coleman, 1991; Smith et al., 2011; De Corato, 2020).

La práctica de los abonos verdes presenta características económicamente viables y un enfoque ambiental positivo, correspondiendo a una alternativa eficiente para la sustitución de los plaguicidas sintéticos (Chimouriya et al., 2018). Los resultados obtenidos concernientes al rendimiento comercial permitirían pensar que es recomendable emplear como abono verde cualquiera de las especies vegetales empleadas en esta investigación. No obstante, de manera práctica se prefiere la cebada por el menor costo comparativo, las posibilidades mayores de consecución de semilla, la mayor plasticidad de la especie por su adaptabilidad en distintos ambientes y el potencial rendidor de biomasa por unidad de superficie. Además de ello, son menores las posibilidades de efectos alelopáticos respecto a las crucíferas (Ramírez et al., 2011; Smith et al., 2011).

\section{CONCLUSIONES}

De los resultados obtenidos y fundamentados en su discusión, se pudieron obtener las siguientes conclusiones: 1) la práctica de empleo y beneficio de abonos verdes condujo a efectos favorables en la productividad de la papa Diacol Capiro con incrementos mayores de las cinco toneladas de producto comercial por hectárea en los ambientes de Pasto, Ipiales y Túquerres; 2) en la etapa de emergencia total, el empleo de avena, cebada, mostaza y nabo reduce la incidencia de $R$. solani respecto al tratamiento testigo en las etapas de emergencia y tuberización; 3) para la etapa de tuberización, se determinó que la cebada fue más efectiva, disminuyendo significativamente la incidencia respecto a los otros abonos verdes empleados; y 4) el efecto benéfico de la cebada produjo incrementos significativos en la producción de tubérculos grandes, razón por la cual se recomienda como la opción más adecuada de abono verde para el manejo de la costra negra en Nariño.

\section{AGRADECIMIENTOS}

Esta investigación es producto del Proyecto de investigación 'Manejo Integrado de la Costra Negra de la papa (Rhizoctonia solani Künh) en el departamento de Nariño' financiado por la convocatoria 818 de Minciencias. 


\section{REFERENCIAS}

Ascencion, L.C., Liang, W.J., y Yen, T.B., Control of Rhizoctonia solani damping-off disease after soil amendment with dry tissues of Brassica results from increase in Actinomycetes population, https://doi.org/10.1016/j.biocontrol.2014.11.010, Bio. Control, 82, 21-30 (2015)

Betancourth, C.A., Sañudo, B.A., y otros cuatro autores, Vulneración del cultivo de papa ante problemas sanitarios emergentes en Nariño, 6-9, Universidad de Nariño, Pasto, Colombia (2020)

Bienkowski, D., Stewart, A., y otros cuatro autores, A disease assay for Rhizoctonia solani on potato (Solanum tuberosum), https://doi.org/10.30843/nzpp.2010.63.6553, N.Z. Plant Prot., 63, 133-137 (2010)

Bonanomi, G., Zotti, M., y otros seis autores, Decomposition and organic amendments chemistry explain contrasting effects on plant growth promotion and suppression of Rhizoctonia solani damping off, https://doi.org/10.1371/journal.pone.0230925, PLoS ONE, 15(4), e0230925 (2020)

Carling, D.E., y Leiner, R.H., Isolation and Characterization of Rhizoctonia solani and Binucleate R. solani-like Fungi from Aerial Stems and Subterranean Organs of Potato Plants, https://doi.org/10.1094/Phyto-76-725, Phytopathology, 76(7), 725-729 (1986)

Chimouriya, S., Lamichhane, J., y Prasad, D., Green Manure for Restoring and Improving the Soil Nutrients Quality, http://edupediapublications.org/journals/index.php/IJR/, Int. J. Res., 5(20), 1064-1074 (2018)

Dayegamiye, A.N., Nyiraneza, J., y otros tres autores, The Benefits of Crop Rotation Including Cereals and Green Manures on Potato Yield and Nitrogen Nutrition and Soil Properties, http://dx.doi.org/10.4172/2329-8863.1000279, Adv. Crop Sci. Tech., 5, 279 (2017)

De Corato, U., Disease-suppressive compost enhances natural soil suppressiveness against soil-borne plant pathogens: A critical review, https://doi.org/10.1016/j.rhisph.2020.100192, Rhizosphere, 13, 100-192 (2020)

Ferrucho, R.L., Ceresini, P.C., y otros cinco autores, The Population Genetic Structure of Rhizoctonia solani AG-3PT from Potato in the Colombian Andes, https://doi.org/10.1094/PHYTO-11-12-0278-R, Phytopathology, 103(8), 862-869 (2013)

Gonzáles, V., Portal, M.A., y Rubio, V., Review. Biology and Systematics of the form Genus Rhizoctonia, https://doi.org/10.5424/sjar/2006041-178, Span. J. Agric. Res., 4(1), 55-79 (2006)

Handiseni, M., Jo, Y.K., y otros dos autores, Screening Brassicaceous Plants as Biofumigants for Management of Rhizoctonia solani AG1-IA, https://doi.org/10.1094/PDIS-06-15-0667-RE, Plant Dis., 100(4), 758-763 (2016)

Khiareddine, H.J., Effect of Fodder Radish (Raphanus sativus L.) Green Manure on Potato Wilt, Growth and Yield Parameters, https://doi.org/10.4172/2329-8863.1000211, Adv. Crop. Sci. Tech., 4(2), 211 (2016)

Kumar, M., Singh, J.K., y otros dos autores, A Comprehensive Overview on Black Scurf of Potato, https://doi.org/10.20546/ijcmas.2017.610.472, Int. J. Curr. Microbiol. App. Sci., 6(10), 498-4994 (2017)

Larkin, R., Green manures and plant disease management, https://doi.org/10.1079/PAVSNNR20138037, CAB Reviews, 8(37) (2013).

Larkin, R.P., y Griffin, T.S., Control of soilborne potato diseases using Brassica green manures, https://doi.org/10.1016/j.cropro.2006.10.004, Crop Prot., 26(7), 1067-1077 (2007)

Larkin, R., y Halloran, J.M., Management Effects of Disease-Suppressive Rotation Crops on Potato Yield and Soilborne Disease and Their Economic Implications in Potato Production, https://doi.org/10.1007/s12230-014-9366-z, Am. J. Potato Res., 91(5), 429-439 (2014)

Manici, L. M., Caputo, F., y Babini, V., Effect of green manure on Pythium spp. population and microbial communities in intensive cropping systems, https://doi.org/10.1023/b:plso.0000047720.40918.29, Plant Soil, 263(1), 133-142 (2004)

Mazzola, M., Management of Resident Soil Microbial Community Structure and Function to Suppress Soilborne Disease Development, in Climate change and crop production by M. Reynolds, 200-218, CAB International, Mexico (2010)

Parkinson, D., y Coleman, D. C., Microbial Communities, Activity and Biomass, https://doi.org/10.1016/01678809(91)90090-K, Agric. Ecosyst. Environ., 4(1-4), 3-33 (1991)

Prager, M., Sanclemente, O.E., y otros tres autores, Abonos verdes: Tecnología para el manejo agroecológico de los cultivos, Agroecología, 7(1), 53-62 (2012)

Ramírez, J., Carrillo, J.M., y otros dos autores, Comparación de gramínea, leguminosa y crucífera para actuar como cubiertas vegetales destinadas a su empleo como abono verde, Actas de Horticultura, 61, 1-7 (2011)

Rubayet, M.T., Bhuiyan, M. K., y otros tres autores, Effect of biofumigation and soil solarization on stem canker and black scurf diseases of potato (Solanum tuberosum L.) caused by Rhizoctonia solani isolate PR2, Advances in Agricultural Science, 6(3), 33-48 (2018)

Sampaio, E.P., Estudio de las Prácticas Culturales en sus Relaciones con Agricultura, Suelo y Ambiente, http://dx.doi.org/10.4067/S0718-07642009000300013, Inf. tecnol., 20 (3), 113-123 (2009) 
Sierotzki, H., y Scalliet, G., A Review of Current Knowledge of Resistance Aspects for the Next-Generation Succinate Dehydrogenase Inhibitor Fungicides, https://doi.org/10.1094/PHYTO-01-13-0009-RVW, Phytopathology, 103(9), 880-887 (2013)

Smith, R., Bugg, R. L., y otros tres autores, Cover Cropping for Vegetable Production: A Grower's Handbook, 41-68, University of California Agriculture and Natural Resources, Richmond, USA (2011)

Soldevilla, M., Influence of Cover Crops on the Development of some Soil-Borne Plant Pathogens, 9-39, Swedish University of Agricultural Sciences, Uppsala, Suecia (2009)

Tsror, L., Biology, Epidemiology and Management of Rhizoctonia solani on Potato, https://doi.org/10.1111/j.14390434.2010.01671.x, J. Phytopathol., 158(10), 649-658 (2010)

Tsror, L., y Peretz A.I., The Influence of the Inoculum Source of Rhizoctonia solani on Development of Black Scurf on Potato, https://doi.org/10.1111/j.1439-0434.2005.00962.x, J. Phytopathol., 153(4), 240-244 (2005)

Vandicke, J., De Visschere, K., y otros cinco autores, Uncovering the biofumigant capacity of allyl isothiocyanate from several Brassicaceae crops against Fusarium pathogens in maize, https://doi.org/10.1002/jsfa.10599, J. Sci. Food Agric., 100(15), 5476-5486 (2020)

Vukicevich, E., Lowery, T., y otros tres autores, Cover crops to increase soil microbial diversity and mitigate decline in perennial agriculture. A review, https://doi.org/10.1007/s13593-016-0385-7, Agron. Sustain. Dev., 36, 48 (2016)

Xiong, L., Shen, Y.Q., y otros cinco autores, Succinate dehydrogenase: An Ideal Target for Fungicide Discovery, in Discovery and Synthesis of Crop Protection Products by P. Maienfisch, y T. Stevenson, 175-194, American Chemical Society, Washington, USA (2015)

Yossen, V., Rojo, R., y otros seis autores, Effect of Green Manure and Biocontrol Agents on Potato Crop in Cordoba, Argentina, J. Plant Pathol., 93(3), 713-717 (2011) 